\title{
Rare, post-periodontitis spondylodiscitis caused by Fusobacterium nucleatum in a patient with multiple sclerosis: challenge of diagnosis and treatment
}

\author{
Dritan Pasku, Siddharth Shah, Ahmed Aly, Nasir A Quraishi
}

The Centre for Spinal Studies and Surgery, Queen's Medical Centre Nottingham, University Hospital NHS Trust, Nottingham, UK

\section{Correspondence to Dritan Pasku;} paskudr@hotmail.com

Accepted 3 March 2021

Check for updates

(c) BMJ Publishing Group Limited 2021. No commercial re-use. See rights and permissions. Published by BMJ.

To cite: Pasku D, Shah $S$, Aly $\mathrm{A}$, et al. BMJ Case Rep 2021;14:e239664. doi:10.1136/bcr-2020239664

\section{SUMMARY}

Fusobacterium nucleatum is part of the commensal flora of the oral cavity, frequently associated with periodontal infections. We describe the case of a 49-year-old woman, on immunsuppressive therapy for multiple sclerosis, who presented with a 3-month history of debilitating back pain. She had a recent episode of periodontitis, and was under regular dental review. Her MRI scan demonstrated findings suggestive of L2-L3 spondylodiscitis. Her CTguided biopsy yielded negative cultures and the patient failed two courses of empirical antibiotic treatment. With clinical and radiological disease progression, she underwent a percutaneous disc washout and biopsy, which subsequently grew F. nucleatum. Treatment with clindamycin and metronidazole was commenced orally for 6 weeks. She improved gradually, and at 1 year follow-up was asymptomatic. The diagnosis of spondylodiscitis caused by $F$. nucleatum is challenging. The perseverance on identification by surgical biopsy, minimally invasive washout and targeted antibiotics are the mainstay of effective treatment.

\section{BACKGROUND}

The last three decades have seen a significant increase in the incidence of pyogenic spondylodiscitis due to a number of multifactorial reasonsmost importantly increased life expectancy, chronic diseases, rise in the prevalence of immunosuppressed patients, intravenous drug abuse and an increase in spinal surgery with instrumentation. ${ }^{1}$ On the other hand, improved radiological investigative sensitivity and techniques with microbiological diagnoses contribute to greater accuracy. ${ }^{12}$ The common causative organisms of spontaneous pyogenic spondylodiscitis are Staphylococcus aureus, Enterococci, Streptococcus species and gram negative bacilli. Only $3 \%-4 \%$ of spondylodiscitis is caused by rare anaerobic pathogens like Propionibacterium acnes and Bacteroides fragilis. ${ }^{2}$

Poor oral hygiene has been traditionally associated with a higher incidence of postoperative spinal infections, ${ }^{3}$ which encompasses gum issues commonly known as 'periodontal disease', normally affecting about $20 \%-50 \%$ of global population. ${ }^{4}$ Recent studies have evidenced a causal association between chronic periodontitis and multiple sclerosis in female patients. ${ }^{5}$ The best treatments in this vulnerable age group are prevention and thorough hygiene, which provide satisfactory results and improve the quality of life. ${ }^{6}$ In patients unable to carry out effective oral hygiene, dental treatment should be performed by dental hygienists and/or dentists to prevent dental caries and periodontal disease. $^{6}$

Fusobacterium nucleatum is an important commensal oral pathogen with a well-defined role in pathogenesis of the periodontal disease. Patients with multiple sclerosis and more importantly, those on immunomodulators, are at a higher risk of developing a spinal infection, which may be caused by atypical pathogens spread haematogenously from the oral source. Such low-virulent organisms present a diagnostic challenge to clinicians and require a keen eye of suspicion, acknowledgement of dental history and more aggressive invasive methods of diagnosis and treatment with surgical biopsy and appropriate antibiotic therapy. We hereby present a rare case of $F$. nucleatum spondylodiscitis in an immunosuppressed patient known to have multiple sclerosis with a background of chronic periodontitis.

\section{CASE PRESENTATION}

A 49-year-old female patient was admitted to a medical ward in our hospital, with chest pain and severe back pain of mechanical nature. The pain was worse while mobilising, with a visual analogue score of 10/10. There was no neurological deficit and her bladder and bowel function was intact. Her body temperature was normal and the blood samples taken on admission showed normal white blood cells, normal liver and renal function; $\mathrm{C}$ reactive protein (CRP) was mildly raised to $48 \mathrm{mg} / \mathrm{L}$ $(0-10 \mathrm{mg} / \mathrm{L})$.

The patient had been diagnosed 8 years previously with multiple sclerosis and had been on dimethyl fumarate $240 \mathrm{mg}$ two times per day (Tecfidera, Biogen, Cambridge, MA, USA) for many years, a disease modifying medication. Further, only 25 days earlier, she had been admitted to another hospital because of severe back pain and fever. The blood cultures taken at previous hospital were negative but her CRP was raised to more than $200 \mathrm{mg} / \mathrm{L}$. An MRI of her lumbar spine showed an infective/ inflammatory process involving the L2/L3 disc space (figure 1). She was treated empirically with intravenous ceftriaxone $2 \mathrm{~g}$ once a day and $220 \mathrm{mg}$ of gentamicin every other day. Her fever, degree of pain and inflammatory markers responded to the treatment. A new MRI before discharge from the previous hospital showed some improvement in the radiological appearance of her infective/ inflammatory process and after 2 weeks of inpatient 


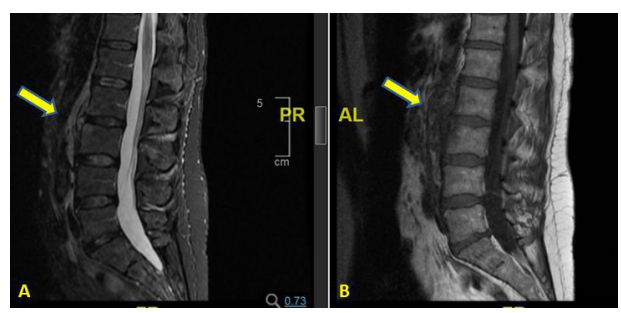

Figure 1 (A) Sagittal T2 STIR (Short-TI Inversion Recovery)-weighted sequence and (B) sagittal T1 sequence of the initial lumbar spine MRI performed at another hospital, showing mild involvement of the $L 2 / 3$ disc space but significant prevertebral collection.

antibiotics she was discharged home with co-amoxiclav (amoxicillin/clavulanic acid) $625 \mathrm{mg}$, three times a day.

As a result of the presence of her chest pain and shortness of breath, she was referred to the respiratory and cardiology teams in our hospital. An echocardiogram revealed of mild pericardial effusion of $0.4 \mathrm{~cm}$ but ruled out the presence of endocarditis. The cardiologists recommended $400 \mathrm{mg}$ of ibuprofen three times a day. However, a lumbar MRI did confirm a worsening radiological picture of the L2/L3 disc space pathology and increased prevertebral collection (figure 2) with a CRP of $16 \mathrm{mg} / \mathrm{L}$. Her case was discussed with the spinal and microbiology teams and a CT-guided biopsy of L2/L3 disc space was recommended (figure 3). Despite a good sample by a senior musculoskeletal radiologist, the result was negative for bacteria, fungi and $\mathrm{Myco-}$ bacterium tuberculosis. Further, the histological examination did not reveal the presence of any infection (including granulomas).

Subsequently, her clinical presentation did improve and she was sent home on ibuprofen as per the advice of the cardiologists without antibiotic therapy because of the lack of evidence of a confirmed infection and only a mildly raised level of CRP to $20 \mathrm{mg} / \mathrm{L}$. Unfortunately, she re-presented 3 weeks later with worsening back pain and this time was admitted to our spinal unit. A new MRI scan showed progression of the L2/L3 spondylodiscitis with bone destruction, an increase in size of the prevertebral collection and bone marrow oedema involving the majority of the L2 and L3 vertebral bodies (figure 4). Her CRP was markedly raised at $62 \mathrm{mg} / \mathrm{L}$ but her white cell count remained normal. This time the decision was to take a more aggressive approach with a percutaneous minimal invasive intervertebral disc biopsy at L2/L3 and washout. Before the procedure, the presence of endocarditis, lung and urinary tract infection or any recent traumatic/invasive procedure was ruled out. F. nucleatum was isolated from the sample and to the patient was administrated clindamycin $450 \mathrm{mg}$ oral four times a day and metronidazole $400 \mathrm{mg}$ three times a day for 6 weeks orally.

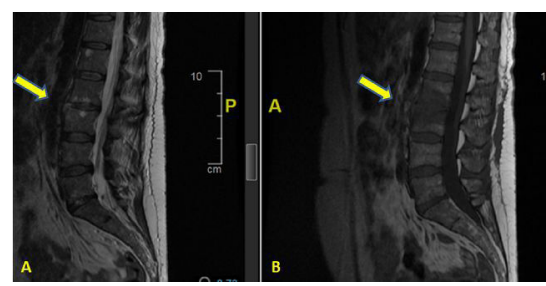

Figure 2 (A) Sagittal T2 STIR(Short-TI Inversion Recovery)-weighted sequence and (B) sagittal T1 sequence of the lumbar spine MRI performed at our hospital before the CT-guided biopsy, showing an increase of the disc involvement as well as expansion of the infective process to the vertebral bodies.

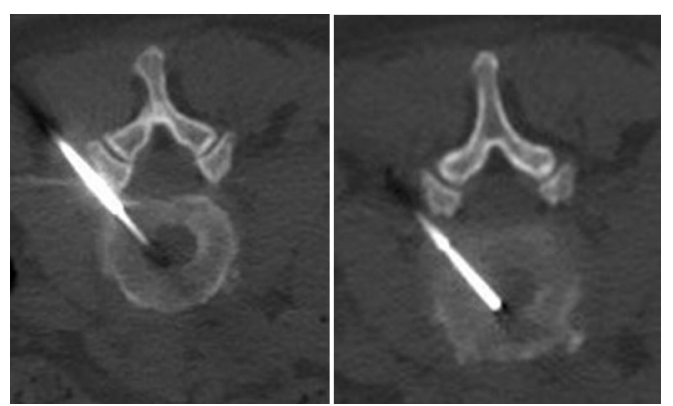

Figure 3 CT-guided biopsy showing optimal positioning of the needle in the involved area of the L2/L3 intervertebral disc.

\section{INVESTIGATIONS}

The combination of the raised CRP value and the radiological investigations were crucial to understand the dynamics of the spinal low-grade infection. The progression from her initial MRI scan (figure 1) performed in another hospital to the subsequent MRI (figure 2) performed in our hospital confirmed disease progression but we believe that the administration of ibuprofen from the cardiologists kept her CRP levels down to $16 \mathrm{mg} / \mathrm{L}$.

From the diagnostic point of view, biopsy is the gold standard procedure to obtain microbiological and histopathological diagnoses in spinal infections. In our opinion, the negative result after a technically correct CT-guided biopsy (figure 3) was likely due to the recent usage of antibiotics as well as to the smaller amount of abscess which reduced bacterial load in the sample.

Fluoroscopic and CT-guided spinal biopsies have been shown to have comparable accuracy ranging from $16.1 \%$ to $92.3 \%$. This variability is probably related to multiple factors such as local anatomy, spinal level, operator experience, biopsy approaches and needle sizes as well as the timing and the accuracy of the microbiological workup. ${ }^{8}$ In a very recent prospective study, the accuracy of spinal biopsy for fluoroscopic-guided spinal biopsy and CT-guided spinal biopsy for spinal infections was similar at 59\% and 61\%, respectively. ${ }^{9}$

The last MRI scan before the surgical treatment scan showed bone destruction, an increase in size of the prevertebral collection progression (figure 4) making the microbiological diagnosis mandatory and the surgical intervention inevitable in order to establish diagnosis and facilitate her treatment.

\section{DIFFERENTIAL DIAGNOSIS}

The presence of back pain with rapidly progressive radiological vertebral end-plate changes is highly suggestive of an infective process in the lumbar spine. ${ }^{12}$ The main differential diagnosis

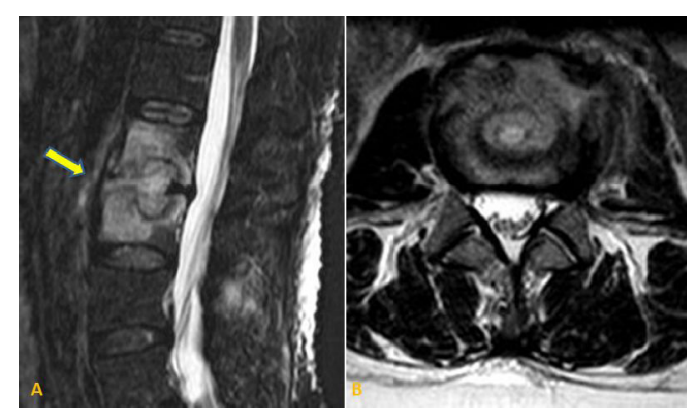

Figure 4 (A) Sagittal T2 STIR(Short-TI Inversion Recovery)-weighted sequence and (B) axial T2 sequence of the preoperative MRI showing an increase in size of the intervertebral abscess and the involvement of almost the entire vertebral bodies of $\mathrm{L} 2$ and $\mathrm{L} 3$. 


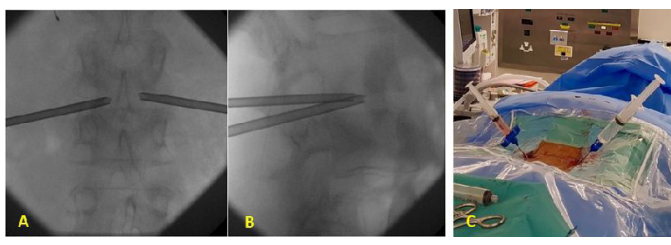

Figure 5 (A) Intraoperative C-arm imaging Antero-Posterior (AP) and (B) lateral projections showing good placement of the Jamshidi needles to the targeted lesion in both antero-posterior and lateral view. (C) Intraoperative picture with good positioning of the Jamshidi needles during the process of washout highlighting the different colours of the syringes due to the saline flow (right needle for injection of saline and left for aspiration).

considerations are aseptic or necrotic changes, but rapid progression combined with the lack of chemotherapy or radiotherapy minimise such a possibility. Thus, the infective process was the main working diagnosis and our challenge was to obtain a good sample and to isolate the causative agent. The history of dental cleaning 4-5 weeks before the onset of the back pain, the negative blood cultures, the low levels of CRP and the fact that the patient had been on long-term immunomodulators and recently on ibuprofen highlighted the possibility of dealing with a rare microbial agent with low virulence, causing atypical spondylodiscitis and even pericardiditis.

\section{TREATMENT}

Our patient underwent a percutaneous minimal invasive intervertebral L2-L3 disc biopsy and disc washout.

Under general anaesthesia, the patient was placed prone on a radiolucent table and the $\mathrm{C}$-arm fluoroscope was positioned to clearly identify the L2-L3 disc space. The discographic approach was used to percutaneously insert two Jamshidi biopsy needles, one either side, directed into the disc space (figure 5A,B). Care was taken to position both needles in the centre of the involved L2-L3 disc. A $20 \mathrm{~mL}$ luer lock syringe was attached to both the Jamshidi needles and used to aspirate the disc content sequentially. The aspirated sample, consisting of a white-yellowish colour high viscosity fluid, was sent for microbiological analysis. The aspirated disc material was sent for histological examination.

Following this, a new $20 \mathrm{~mL}$ syringe containing normal saline was attached to the Jamshidi needle on one side and an empty syringe on the opposite side. The disc was then washed out with normal saline solution from one needle, which was aspirated and collected in the empty syringe on the opposite side (figure 5C). The first $10 \mathrm{~mL}$ of saline disc washout was sent for an additional microbiological evaluation, and the remaining washout fluid was discarded. Interestingly, active suction with the empty syringe was not required as the injected saline pressure from one side filled up the empty syringe on the other side. This process was then repeated and reversed until the fluid ran clear and at least $500 \mathrm{~mL}$ of normal saline has been flushed through the disc space. The needles were withdrawn, the stab incisions were closed with 3-0 ethilon sutures and sterile dressings applied. There were no perioperative surgical complications.

This technique (not too dissimilar to a discography approach) can be performed under local anaesthesia or conscious sedation and is very cost effective as only a C-arm image intensifier and two Jamshidi needles are required. The access to the disc space through soft tissue provides more freedom and better positioning of the needle to the disc space, allowing debridement of the disc space and decrease of the bacterial load through the continuous washout. The aspiration on one side is performed while saline is injected from the opposite side. Further, the discographic approach avoids the penetration of the bone elements of the posterior arch and the spinal canal, reducing the risk of posterior spread of the infection and other complications. If considered appropriate, local antibiotics can be injected at the end of the procedure. Finally, this simple technique is technically less demanding and can be easily adapted to different hospital settings. ${ }^{10}$

The microbiological samples isolated F. nucleatum and based on the drug sensitivity patterns, with advice from the microbiology team, the patient was started on clindamycin $450 \mathrm{mg}$ orally four times a day and metronidazole $400 \mathrm{mg}$ orally three times a day for 6 weeks.

\section{OUTCOME AND FOLLOW-UP}

Immediately after the surgery, the patient's pain improved by more than $50 \%$. The CRP decreased to half its preoperative value 2 days after the procedure, and was back to normal at 2 weeks postoperatively. She was discharged from the hospital on oral antibiotics. She was subsequently seen regularly at 6 weeks, 6 months and a year after the procedure. Her symptoms gradually improved, with no back pain and her inflammatory markers all remained within the normal range throughout the course of treatment and follow-up. A follow-up MRI performed at the end of the 1 year follow-up (figure 6) showed excellent resolution of the infection, with only a minimal residual marrow oedema; our patient was entirely asymptomatic and discharged from the spinal team.

\section{DISCUSSION}

We speculated that, during the process of professional dental cleaning, our patient sustained some bleeding from her gum and this most likely caused a temporary bacteremia, which was then propagated to a distant intervertebral space. In fact, her back pain started 4-5 weeks after the oral cleansing. Moreover, the patient suffered from a concomitant pericarditis and is not unlikely that, both her spondylodiscitis and pericarditis to have been caused by the same pathogen. The pericarditis caused by anaerobic bacteria is a rare but well-known entity with the first case of pericarditis caused by Fusiform nucleatum reported by Truant et al. ${ }^{1112}$

Our patient was on dimethyl fumarate (Tecfidera, Biogen, USA), a pleiotropic immunomodulatory medication of the immune system. The main adverse effect is the reduction in the amount of lymphocytes, although only about 4\% (1 in 25) of patients treated with Tecfidera develop a more serious drop in lymphocyte count, resulting in lymphopenia. The most serious adverse effect are progressive multifocal leukoencephalopathy and Fanconi syndrome, which are considered very rare. ${ }^{13}$ Dimethyl fumarate which had not caused lymphopenia, but in any case was stopped after the suspicion of infection.

More than 700 bacterial species or phylotypes, of which over 50\% have not been cultivated, have been detected in the oral cavity. ${ }^{14}$ Among them, Fusobacterium is a genus of anaerobic filamentous gram-negative rods that are members of the phylum Fusobacteria. They can be divided into 13 species that inhabit the oral, gastrointestinal, upper respiratory tract and vaginal mucosa. F. nucleatum is the species most commonly described causing human infection and Lemierre's syndrome is the best known infection associated with it. ${ }^{15}$ Although, recently, Laurencet $e t$ al, reported a case of Lemierre's syndrome caused by F. nucleatum and co-existing L4/L5 spondylodiscitis. ${ }^{16}$ 


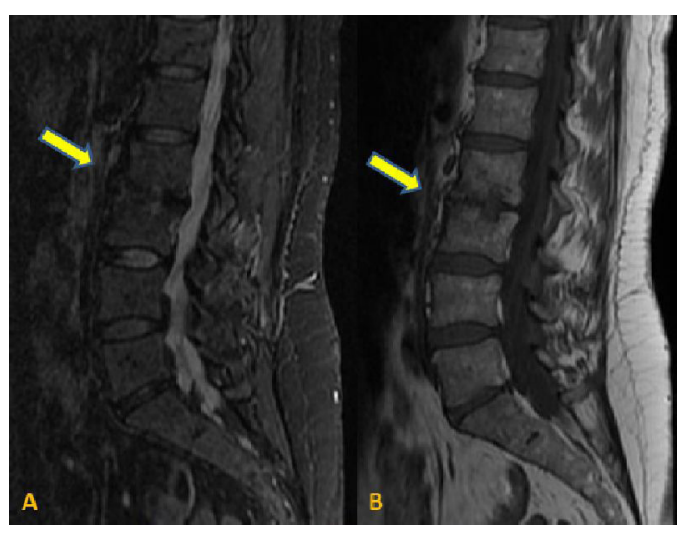

Figure 6 (A) Sagittal T2 STIR(Short-TI Inversion Recovery)-weighted and (B) sagittal T1 sequence of the lumbar spine MRI performed one year postoperatively, showing excellent response with significant resolution of the reactive oedema and improvement of the infective process affecting the L2/L3 intervertebral disc.

To our knowledge, only 18 cases of vertebral spondylodiscitis due to the Fusobacterium species have been described in the literature. ${ }^{16-18}$ Of those, 12 cases were exclusively caused by F. nucleatum. In approximately $1 / 3$ of cases, the diagnosis was based on the blood cultures and in $2 / 3$ of the cases on disc aspirates. All samples required prolonged incubation at least 3 days but in one case, PCR followed by $16 \mathrm{~S}$ rDNA sequencing was required to detect the pathogen. ${ }^{18}$ Seven of 18 patients detected

\section{Learning points}

- Fusobacterium nucleatum, although a normal commensal of oral flora, can cause pyogenic spondylodiscitis especially in susceptible immunocompromised population.

- Taking an accurate history of oral hygiene and possible dentistry procedures is crucial when dealing with the possibility of distant infections such as spondylodiscitis and cardiac infections.

- Accurate microbiological diagnosis and drug-sensitive antibiotic therapy are effective in treating infection in the absence of neurological symptoms.

- CT-guided biopsy may be insufficient in providing adequate sample to isolate the pathogen and a low threshold for a surgical biopsy should be maintained. This enables an adequate sample for microbiological and histopathological diagnoses to be obtained, reduces the bacteriological load providing immediate postoperative relief of symptoms and improves the antibiotic penetration and efficacy.

- The importance of a multidisciplinary team effort and awareness is highlighted. Dentists should be aware of the risk of haematogenous spread of commensal oral flora in patients suffering with periodontitis; neurologists should recognise the possibility of atypical distant systemic infections in patients with multiple sclerosis on immunomodulators; microbiologists should maintain a high index of suspicion for low-grade atypical infective pathogens and ensure adequate culture techniques are employed to isolate these organisms; lastly, spinal surgeons should persist in obtaining an accurate sample from the infective site, and in the presence of lowvirulent infections, adopt a more aggressive approach with minimally invasive methods of percutaneous disc biopsy and washout. in the literature $(39 \%)$ had significant history of oral problems: five with periodontitis and two with dental root abscess. In some cases the oral pathology was completely asymptomatic and the correlation between the spinal infection and oral flora was made after the cultures. ${ }^{17}$

The hallmark in the successful management of spinal spondylodiscitis is the isolation of the causative agent. Medical treatment remains the first-line management but the presence of neurological compromise with or without vertebral collapse and failure of conservative management are the two main indications for surgical treatment.

In the majority of cases, the treatment of the spondylodiscitis caused by $F$. nucleatum is medical, with a combination of antibiotics. In only 4 out of 18 cases, the treatment was surgical with open surgery to drain the abscess and/or to stabilise the spine performed. $^{16-18}$

We preferred the minimal invasive percutaneous biopsy and washout because of several reasons. ${ }^{19}$ First, the amount of the pus in the most recent MRI was greater, giving us a confidence in achieving a good representative sample. Second, in immunocompromised patients, a minimal access approach is advantageous to reduce the chances of postoperative complications associated with larger (open) surgeries. Finally, alongside the biopsy, we performed surgical irrigation and debridement in order to maximise the treatment.

Acknowledgements The authors are particularly grateful to Dr Vivienne Weston, consultant microbiologist and Dr Yuriy Arlachov, consultant radiologist for their crucial help throughout the treatment of this challenging case. Many thanks to the patient for the support given to facilitate the publication of this interesting case.

Contributors DP initiated the data collection and designated the work, wrote the manuscript and contributed to surgical management. SS and AA co-wrote the manuscript and contributed in the follow-up of the patient. NAQ performed the critical revision of the article and final approval of the version to be published.

Funding The authors have not declared a specific grant for this research from any funding agency in the public, commercial or not-for-profit sectors.

Competing interests None declared.

Patient consent for publication Obtained.

Provenance and peer review Not commissioned; externally peer reviewed.

\section{REFERENCES}

1 Pola E, Taccari F, Autore G, et al. Multidisciplinary management of pyogenic spondylodiscitis: epidemiological and clinical features, prognostic factors and longterm outcomes in 207 patients. Eur Spine J 2018;27:229-36.

2 Gouliouris T, Aliyu SH, Brown NM. Spondylodiscitis: update on diagnosis and management. J Antimicrob Chemother 2010;65:11:iii11-24.

3 Romagna A, Troeltzsch M, Birkenmaier C, et al. Oral cavity infection: an underestimated source of pyogenic spondylodiscitis? J Neurol Surg A Cent Eur Neurosurg 2018;79:218-23.

4 Nazir MA. Prevalence of periodontal disease, its association with systemic diseases and prevention. Int J Health Sci 2017;11:72-80.

5 Sheu J-J, Lin H-C. Association between multiple sclerosis and chronic periodontitis: a population-based pilot study. Eur J Neurol 2013;20:1053-9.

6 Elemek E, Almas K. Multiple sclerosis and oral health: an update. NY State Dent J 2013;79:16-21.

7 Rimondi E, Staals EL, Errani C, et al. Percutaneous CT-guided biopsy of the spine: results of 430 biopsies. Eur Spine J 2008;17:975-81.

8 Heyer CM, Brus L-J, Peters SA, et al. Efficacy of CT-guided biopsies of the spine in patients with spondylitis--an analysis of 164 procedures. Eur J Radiol 2012;81:e244-9.

9 Lee SA, Chiu CK, Chan CYW, et al. The clinical utility of fluoroscopic versus CT guided percutaneous transpedicular core needle biopsy for spinal infections and tumours: a randomized trial. Spine J 2020;20:1114-24.

10 Griffith-Jones W, Nasto LA, Pola E, et al. Percutaneous suction and irrigation for the treatment of recalcitrant pyogenic spondylodiscitis. J Orthop Traumatol 2018;19:20:10.

11 Brook I. Pericarditis caused by anaerobic bacteria. Int J Antimicrob Agents 2009;33:297-300. 
12 Truant AL, Menge S, Milliorn K, et al. Fusobacterium nucleatum pericarditis. J Clin Microbiol 1983;17:349-51.

13 Klotz L, Havla J, Schwab N, et al. Risks and risk management in modern multiple sclerosis immunotherapeutic treatment. Ther Adv Neurol Disord 2019;12:12.

14 Aas JA, Paster BJ, Stokes LN, et al. Defining the normal bacterial flora of the oral cavity. J Clin Microbiol 2005:43:5721-32.

15 Goldberg EA, Venkat-Ramani T, Hewit M, et al. Epidemiology and clinical outcomes of patients with Fusobacterium bacteraemia. Epidemiol Infect 2013;141:325-9.

16 Laurencet M-E, Rosset-Zufferey S, Schrenzel J. Atypical presentation of Lemierre's syndrome: case report and literature review. BMC Infect Dis 2019;19:21:868.
17 Latta TN, Mandapat AL, Myers JP. Anaerobic spondylodiscitis due to Fusobacterium species: a case report review of the literature. Case Rep Infect Dis 2015;2015:759539.

18 Mediavilla-Santos L, Fernández-Mariño JR, Sánchez-Somolinos M, et al. [Spondylodiscitis due to Fusobacterium nucleatum: new diagnostic method]. Acta Ortop Mex 2014;28:248-52.

19 Hadjipavlou AG, Katonis PK, Gaitanis IN, et al. Percutaneous transpedicular discectomy and drainage in pyogenic spondylodiscitis. Eur Spine J 2004;13:707-13.

Copyright 2021 BMJ Publishing Group. All rights reserved. For permission to reuse any of this content visit https://www.bmj.com/company/products-services/rights-and-licensing/permissions/

BMJ Case Report Fellows may re-use this article for personal use and teaching without any further permission.

Become a Fellow of BMJ Case Reports today and you can:

- Submit as many cases as you like

- Enjoy fast sympathetic peer review and rapid publication of accepted articles

- Access all the published articles

Re-use any of the published material for personal use and teaching without further permission

Customer Service

If you have any further queries about your subscription, please contact our customer services team on +44 (0) 2071111105 or via email at support@bmj.com.

Visit casereports.bmj.com for more articles like this and to become a Fellow 